EPJ Web of Conferences 65, 02002 (2014)

DOI: $10.1051 /$ epjconf/ 20146502002

(C) Owned by the authors, published by EDP Sciences, 2014

\title{
Semi-empirical calculation of quenching factors for scintillators: new results
}

\author{
V.I. Tretyak ${ }^{1}$ \\ ${ }^{1}$ Institute for Nuclear Research, MSP 03680 Kyiv, Ukraine
}

\begin{abstract}
New results of calculation of quenching factors for ions in scintillators in semi-empirical approach described in [1] are presented. In particular, they give additional arguments in favour of hypothesis that quenching factors for different particles can be described with the same Birks factor $k B$, if all the data were collected in the same conditions and processed in the same way.
\end{abstract}

\section{Introduction}

It has long been experimentally known [2] that light yield $(L)$ of scintillators for incoming heavy particles (protons, $\alpha$ particles, heavier ions) is lower than that for electrons of the same energy: $L_{i}=Q F \cdot L_{e}$, where $Q F$ is the quenching factor. It depends on many conditions: (1) scintillating material itself (with specific impurities and defects); (2) type and quantity of dopants (if any); (3) temperature; (4) type of particle ( $p, \alpha$, heavier ions); (5) particle's energy; (6) electric fields (if any); (7) conditions of measurements (including e.g. time of collection of scintillation signals because different particles produce faster or slower scintillation response). Because of these dependencies, we do not consider $Q F$ (and related Birks factor $k B$, see later) as some fundamental constant of a given scintillating material (as it is quite often considered) but as a variable associated with specific set-up, conditions of measurement and data accumulation and processing. The $Q F$ values are usually in the range from $\simeq 0.8$ for protons to $\simeq 0.02$ for heavy ions (e.g. W). More details and examples can be found in [1].

Knowledge of quenching factors is very important in sensitive searches for Weakly Interacting Massive Particles (WIMPs) [3] through their scattering on atomic nuclei incorporated in massive scintillators or scintillating bolometers installed deep underground, like in the DAMA/LIBRA dark matter (DM) experiment with 250 $\mathrm{kg}$ of $\mathrm{NaI}(\mathrm{Tl})$ [4]. But, generally speaking, information on $Q F$ values is necessary in any measurement of signals from ions with the help of scintillators.

To measure quenching factors, ion's beams are used (see e.g. [5]), or monoenergetic neutron sources (e.g. [6]) which allow to create nuclear recoils with known energies in a bulk of a scintillator. Theoretical models were developed in papers [7-10] but any of them does not allow to predict $Q F$ for all detectors and for any particle at any energy (and very often even to describe already measured experimental data).
In these circumstances, some semi-empirical approaches, which allow to describe measured data and predict the needed $Q F$ 's, are valuable. One of the methods, built on semi-empirical formula of Birks [2], was presented in [1]. Being quite simple and based on publicly available software, it nevertheless allowed to successfully describe $Q F$ 's for many measurements with scintillators of different kinds. Here we present new results obtained after publication of Ref. [1].

\section{Outlines of the method}

Quenching factor for ion is calculated as a ratio of light yield for ion to that for electrons:

$$
Q F_{i}(E)=\frac{L_{i}(E)}{L_{e}(E)}=\frac{\int_{0}^{E} \frac{d E}{1+k B\left(\frac{d E}{d r}\right)_{i}}}{\int_{0}^{E} \frac{d E}{1+k B\left(\frac{d E}{d r}\right)_{e}}},
$$

where $(d E / d r)_{i}$ and $(d E / d r)_{e}$ are stopping powers (SP) for ions and electrons, respectively, and $k B$ is the so-called Birks factor. It is clear that the result depends on approximations used in calculations of $(d E / d r)_{i}$ and $(d E / d r)_{e}$. Stopping powers for ions are calculated here with the SRIM code [11], and for electrons with the ESTAR program [12]. Both codes are publicly available, easy to use and, in fact, present one of the best software in the field. In both cases, total stopping powers are used for $Q F$ calculations.

It is possible to obtain the following approximation (see [1]) for the quenching factor:

$$
Q F_{i}(E) \simeq \frac{1}{k B(d E / d r)_{i}}
$$

which demonstrates that $Q F_{i}$ depends on energy and is minimal when $(d E / d r)_{i}$ is maximal.

In the approach presented above, quenching factors depend only on one parameter $k B$ which can be found from 


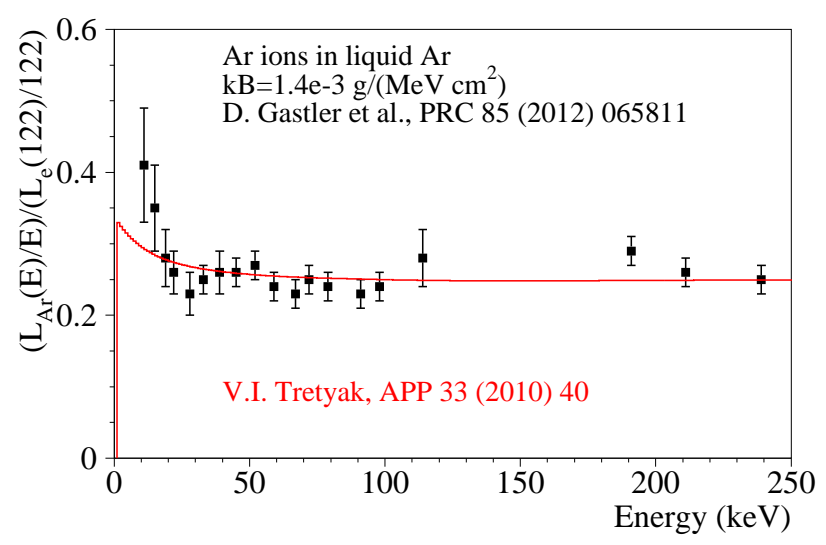

Figure 1. Quenching factors for Ar ions in liquid Ar: experimental data [6] together with calculations with Eq. (1).

fit of some experimental $Q F$ data. This has as some demerits, because bigger number of parameters usually allow to describe experimental data in a better way, but also merits, because in ideal case only one experimental point allows to find the $k B$ value and after to calculate $Q F$ with this $k B$ for all other particles and all energies of interest.

\section{Results}

Below we present some new results obtained after publication of work [1].

(1) Ar ions in liquid Ar. Quenching factors for Ar ions in liquid Ar were measured in the MicroCLEAN studies in [6] in the energy range $11-239 \mathrm{keV}$ (see Fig. 1). The data at energies above $20 \mathrm{keV}$ were approximated in [6] just by constant value $Q F=0.25$, and it was written that "An observed upturn in the scintillation efficiency below $20 \mathrm{keVr}$ is currently unexplained." Fig. 1 shows also curve calculated with Eq. (1) with the value $k B=1.4 \mathrm{mg} /\left(\mathrm{MeV} \cdot \mathrm{cm}^{2}\right)$; one can see that it describes also the upturn below $20 \mathrm{keV}$, not ideally but better than just a constant.

Quenching factors for Ar ions in liquid Ar were also measured recently inside the DARWIN framework [13]. They are shown in Fig. 2a together with data [6] and two theoretical models of Lindhard [8] and Mei [10]. Fig. 2b compares these data with calculations in accordance with Eq. (1) with $k B=1.25 \mathrm{mg} /\left(\mathrm{MeV} \cdot \mathrm{cm}^{2}\right)$.

(2) Alpha particles in $\mathrm{CdWO}_{4}$ scintillating bolometer. $Q F$ values for $\alpha$ particles in $\mathrm{CdWO}_{4}$ bolometer were measured in [14] using internal trace contaminations in the range of $2.5-4.9 \mathrm{MeV}$. They are shown in Fig. 3a, together with the curve calculated with Eq. (1) and $k B=8.8$ $\mathrm{mg} /\left(\mathrm{MeV} \cdot \mathrm{cm}^{2}\right)$. The light yield obtained in a wider energy range with a smeared $\alpha$ source facing $\mathrm{CdWO}_{4}$ is shown in Fig. $3 \mathrm{~b}$ (experimental points vs present calculations).

(3) Protons, $\alpha$ particles, $\mathrm{Li}, \mathrm{C}, \mathrm{O}, \mathrm{Ti}$ ions in $\mathrm{CdWO}_{4}$ crystal scintillator. $Q F$ values for $p, \alpha$, and $\mathrm{Li}, \mathrm{C}, \mathrm{O}$, Ti ions were recently measured with $\mathrm{CdWO}_{4}$ scintillator and ion's beams with $1-10 \mathrm{MeV}$ energies produced by the Tandetron accelerator of LABEC at the INFNFlorence [5]. They are shown in Fig. 4. The data for protons were used to determine the $k B$ value as $k B=17.4$
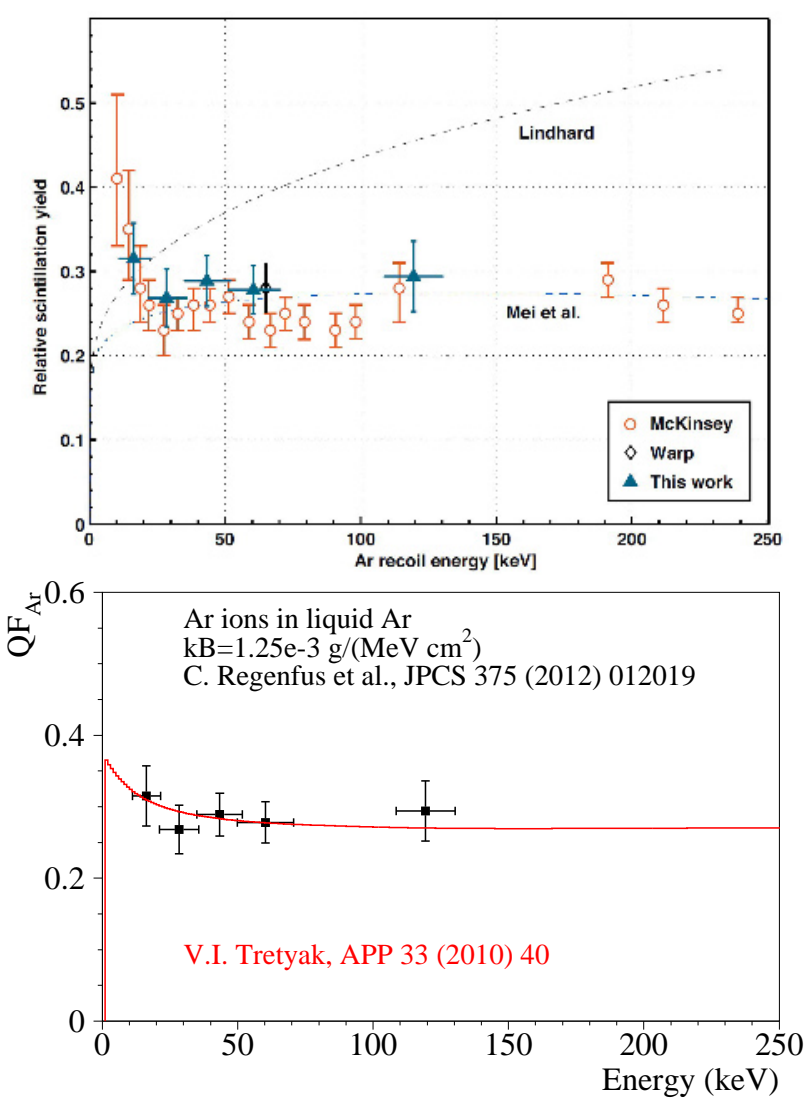

Figure 2. Quenching factors for Ar ions in LAr: (top) experimental data $[6,13]$ together with theoretical curves $[8,10]$ (Fig. 8 of Ref. [13]); (bottom) data [13] together with curve calculated with Eq. (1).
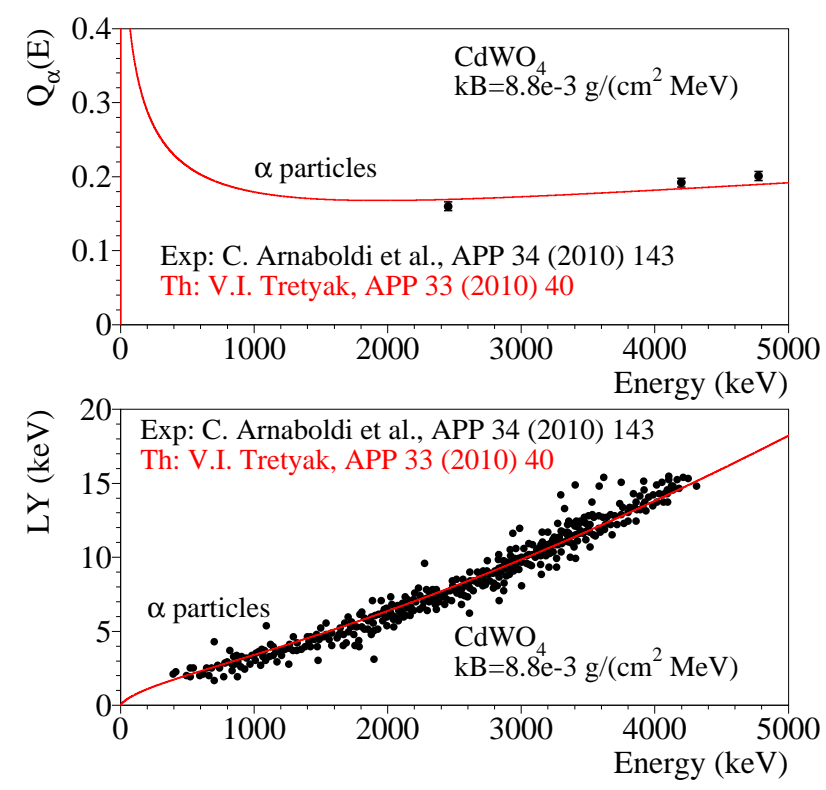

Figure 3. Quenching factors for $\alpha$ particles in $\mathrm{CdWO}_{4}$ scintillating bolometer: (top) experimental $Q F$ 's [14] together with fitting curve; (bottom) the light yields measured with a smeared $\alpha$ source: experimental points [14] and curve calculated with $k B=8.8 \mathrm{mg} /\left(\mathrm{MeV} \cdot \mathrm{cm}^{2}\right)$. 


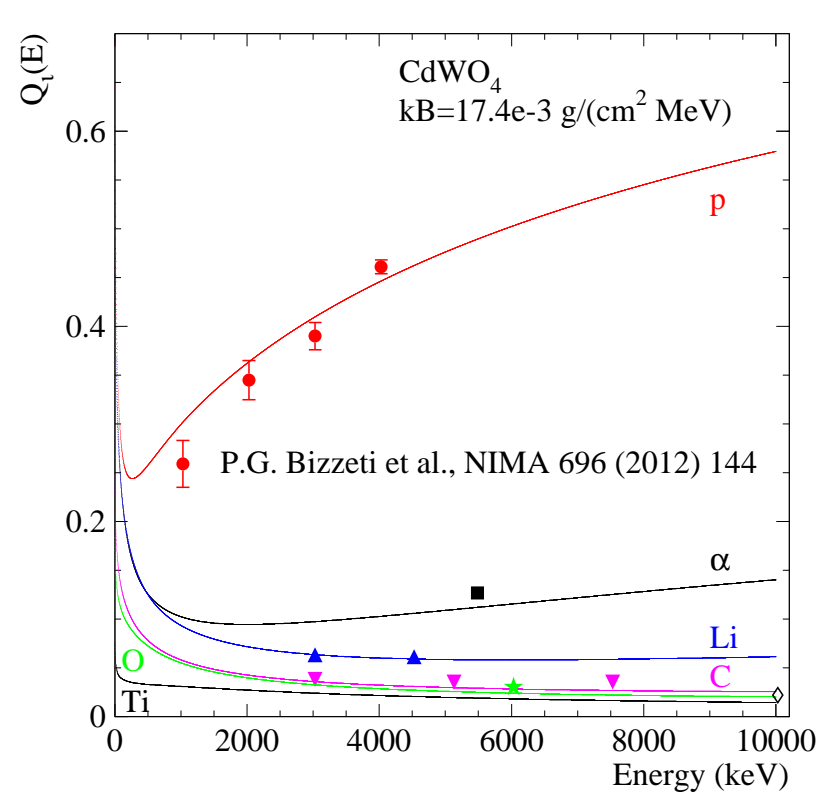

Figure 4. Quenching factors for $p, \alpha$ particles, $\mathrm{Li}, \mathrm{C}, \mathrm{O}$ and Ti ions measured with the $\mathrm{CdWO}_{4}$ crystal scintillator [5]. Solid lines represent calculations with Eq. (1).

$\mathrm{mg} /\left(\mathrm{MeV} \cdot \mathrm{cm}^{2}\right)$, and after curves for all other ions were calculated with this $k B$. Fig. 4 shows general agreement between the experimental results and theoretical dependencies. The biggest deviation is $\simeq 30 \%$ for Ti ions, what could be accepted as satisfactory, especially for theory with one parameter. It should be also noted that sometimes $Q F$ 's in WIMP searches are known with bigger uncertainties, and thus the present approach could give useful information on the expected values of $Q F$ 's.

We would like also to draw attention to difference in quenching factors for $\alpha$ particles in $\mathrm{CdWO}_{4}$ in Fig. 3 and Fig. 4 (that results also in $k B$ values which are different by factor of $\simeq 2$ ). This is one of demonstrations of the statement given in the Introduction: $Q F$ 's (and related $k B$ ) values depend on experimental conditions and cannot be considered as some fundamental constants for a given scintillating material.

(4) Alpha particles in plastic scintillator $\mathrm{C}_{8} H_{8}$. Quenching factors for $\alpha$ particles in solid plastic scintillator $\mathrm{C}_{8} \mathrm{H}_{8}(\mathrm{BiPo}$, in $\mathrm{R} \& \mathrm{D}$ for future SuperNEMO experiment to search for neutrinoless double beta decay) were measured in [15]. They are presented in Fig. 5; calculated curve of $1 / Q F$ with $k B=8.5 \mathrm{mg} /\left(\mathrm{MeV} \cdot \mathrm{cm}^{2}\right)$ is in good agreement with these data.

(5) Protons in pseudocumene $C_{9} H_{12}$. In our previous work [1], quenching factors for $\alpha$ particles in liquid scintillator $\mathrm{C}_{9} \mathrm{H}_{12}$ obtained in the BOREXINO experiment [16] were compared with calculations with Eq. (1) (see Fig. 3c of Ref. [1]). Good agreement was found for the value of $k B=9.4 \mathrm{mg} /\left(\mathrm{MeV} \cdot \mathrm{cm}^{2}\right)$. Later (after publication of [1]) also $Q F$ 's for protons were measured at two energies [17]. Corresponding theoretical curve, calculated with the same $k B$ value, is presented in Fig. 6 . As one can see, agreement is excellent, and this gives additional argument in favour of possibility to describe quenching factors for different

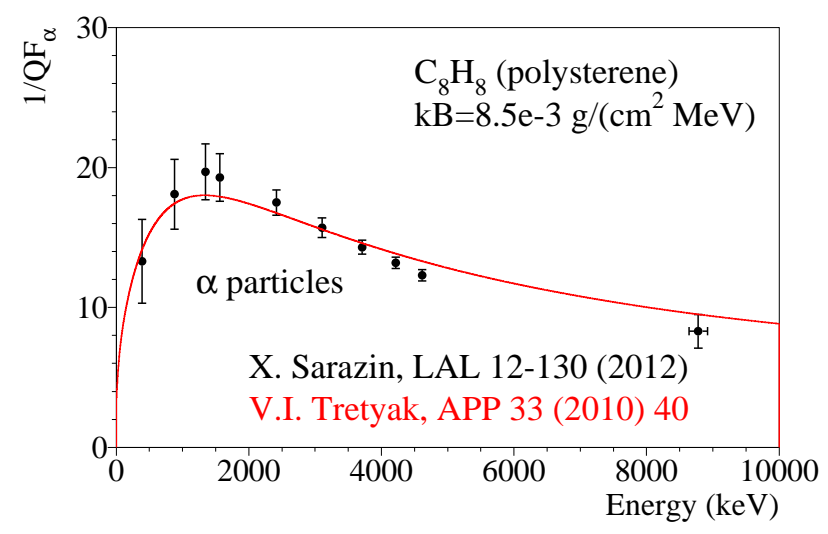

Figure 5. Quenching factors for $\alpha$ particles in $\mathrm{C}_{8} \mathrm{H}_{8}$ scintillator [15]. Solid line represents calculations with Eq. (1).

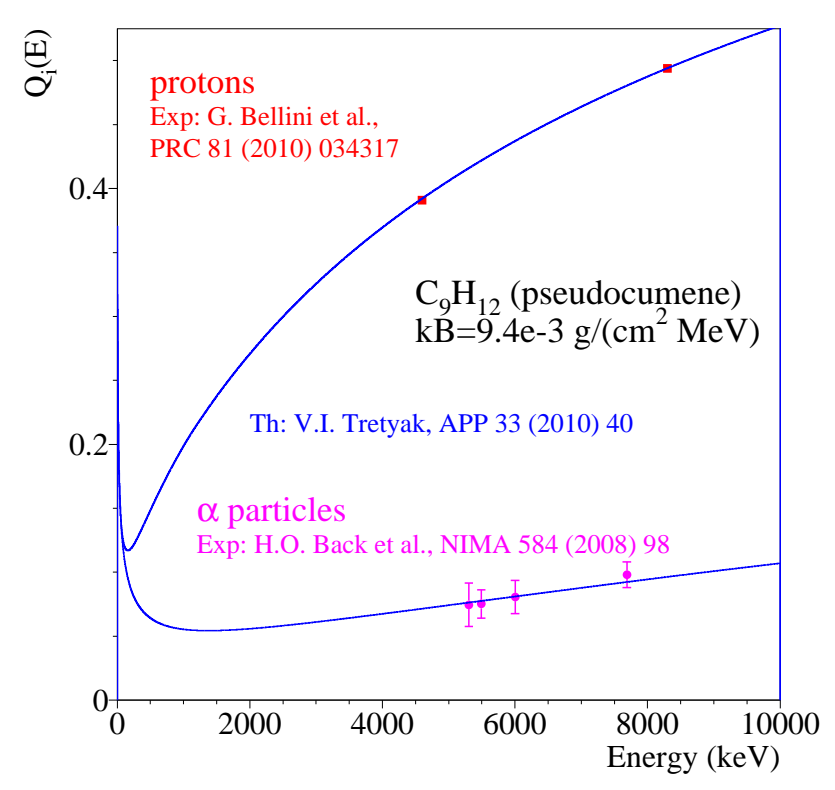

Figure 6. Experimental quenching factors for $\alpha$ particles [16] and protons [17] in pseudocumene $\mathrm{C}_{9} \mathrm{H}_{12}$ liquid scintillator together with calculated curves.

particles with the same $k B$ value if $Q F$ 's are measured in the same conditions.

(6) Quenching factors for $\mathrm{Na}$ and I recoils in the $D A M A$ experiment. While we do not present here new calculations of $Q F$ 's for $\mathrm{Na}$ and I recoils in the DAMA experiment, we would like to comment once more results obtained already in [1]. In the DAMA paper [18], quenching factors for $\alpha$ particles were derived using internal trace contamination of the DAMA NaI(Tl) scintillators by $\mathrm{U} / \mathrm{Th}$ chains. These $Q F_{\alpha}$ 's were obtained in the same conditions as the DAMA dark matter data were accumulated. Fit of the $Q F_{\alpha}$ 's by Eq. (1) allowed to obtain the value $k B=1.25$ $\mathrm{mg} /\left(\mathrm{MeV} \cdot \mathrm{cm}^{2}\right)$ and calculate with this value curves for $\mathrm{Na}$ and I ions. This gave results $Q F_{N a} \simeq 0.65$ and $Q F_{I} \simeq 0.35$ at energies $2-6 \mathrm{keV}$, much higher than the "standard" values of $Q F_{N a}=0.25-0.40$ and $Q F_{I}=0.05-0.10$ used in $\mathrm{NaI}(\mathrm{Tl}) \mathrm{DM}$ experiments (see [1] for corresponding references). However, Figs. 4 and 6 here (together 


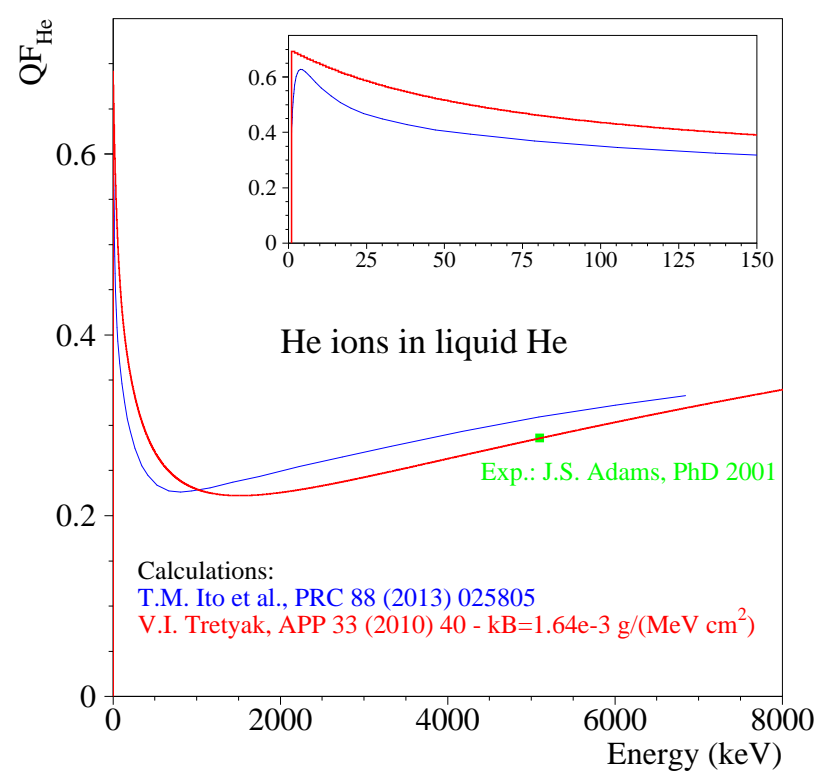

Figure 7. Quenching factors for $\alpha$ particles in liquid He calculated in [23] (dashed curve) and in the present approach (solid curve, normalized to experimental point of [24]).

with Figs. 3d, 4b, 10a, 10b\&c, 13a in [1]) give arguments that $Q F$ 's for $\mathrm{Na}$ and I recoils could be really higher in the DAMA measurements. Higher quenching factors lead to shift of WIMPs mass in the DAMA measurements to lower values of $\simeq 10 \mathrm{GeV}$ (see e.g. [19-21]).

It is also interesting to note that in works [22], where a combined fit of evidences for DM particles in the DAMA, CoGeNT, CRESST, and CDMS-II-Si experiments is described, one of the procedures allows $Q F_{N a}$ to be a free parameter in the range of $0.2-0.6$. In this case fit prefers value of $\simeq 0.6$, and possibly even higher value could be preferred if wider range would be allowed.

(7) Alpha particles in liquid He. Laborious analysis of scintillation yields for electrons and He recoils in $\mathrm{LHe}$ was performed recently in Ref. [23] for possible use of LHe as a detector in DM experiments. The results are shown in Fig. 7. We present also a curve obtained in much simpler semi-empirical calculations with Eq. (1) which is normalised on single experimental point measured in [24]. Both the curves are in satisfactory agreement (taking also into account that uncertainties in calculations of [23] are estimated as $30 \%$ at low energies).

\section{Conclusions}

New results of calculation of quenching factors for ions in scintillators in semi-empirical approach described in [1] are presented here. The old Birks formula still gives nice description of $Q F$ 's for ions in many cases, if the total stopping powers for electrons and ions are used, and SP are calculated with the ESTAR and SRIM codes which: (a) are publicly available, (b) are ones of the best codes in this field. There is only one free parameter in the approach: the Birks $k B$ factor. It is not considered as some fundamental constant for a given scintillating material but as a variable which depends on conditions of measurements and data treatment.

The results presented here give additional arguments in favour of the hypothesis that, once conditions of measurements and data treatment are fixed, the $k B$ value is the same for different ions. Thus, if $k B$ was determined by fitting data for particles of one kind, it can be used to calculate $Q F$ 's for particles of another kind and for another energies of interest.

Quenching factors for ions calculated in the present approach in general increase at low energies, making experimental searches for DM particles more sensitive to low energies and low WIMPs masses.

\section{References}

[1] V.I. Tretyak, Astropart. Phys. 33, 40 (2010)

[2] J.B. Birks, Proc. Phys. Soc. A 64, 874 (1951); The Theory and Practice of Scintillation Counting (Pergamon Press, Oxford, 1964)

[3] K. Freese et al., Rev. Mod. Phys. 85, 1561 (2013)

[4] R. Bernabei et al., Int. J. Mod. Phys. A 28, 1330022 (2013)

[5] P.G. Bizzeti et al., Nucl. Instrum. Meth. A 696, 144 (2012)

[6] D. Gastler et al., Phys. Rev. C 85, 065811 (2012)

[7] R.B. Murray, A. Meyer, Phys. Rev. 122, 815 (1961)

[8] J. Lindhard et al., Mat. Fys. Medd. K. Dan. Vidensk. Selsk. 33, 1 (1963)

[9] A. Hitachi, Astropart. Phys. 24, 247 (2005); J. Phys.: Conf. Ser. 65, 012013 (2007)

[10] D.-M. Mei et al., Astropart. Phys. 30, 12 (2008)

[11] J.F. Ziegler et al., http://www.srim.org

[12] M.J. Berger et al., http://physics.nist.gov/PhysRefData/Star/Text/contents.html

[13] C. Regenfus et al., J. Phys.: Conf. Ser. 375, 012019 (2012)

[14] C. Arnaboldi et al., Astropart. Phys. 34, 143 (2010)

[15] X. Sarazin, Memoire d'habilitation a diriger les recherches, LAL 12-130, Orsay (2012)

[16] H.O. Back et al., Nucl. Instrum. Meth. A 584, 98 (2008)

[17] G. Bellini et al., Phys. Rev. C 81, 034317 (2010)

[18] R. Bernabei et al., Nucl. Instrum. Meth. A 592, 297 (2008)

[19] P. Belli et al., Phys. Rev. D 84, 055014 (2011)

[20] C. Kelso et al., Phys. Rev. D 85, 043515 (2012)

[21] S. Scopel et al., Phys. Rev. D 88, 023506 (2013)

[22] C. Arina et al., JCAP 09, 022 (2011); J. Phys.: Conf. Ser. 375, 012009 (2012); arXiv:1310.5718 [hep-ph] (2013)

[23] T.M. Ito, G.M. Seidel, Phys. Rev. C 88, 025805 (2013)

[24] J.S. Adams, PhD thesis, Brown University (2001) 